Pacific Journal of Mathematics

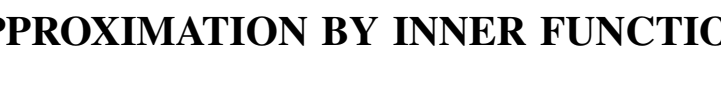




\section{APPROXIMATION BY INNER FUNCTIONS}

\section{R. G. Douglas AND Walter Rudin}

Let $L^{\infty}(T)$ denote the complex Banach algebra of (equivalence classes of) bounded measurable functions on the unit circle $T$, relative to Lebesgue measure $m$. The norm $\|f\|_{\infty}$ of an $f$ in $L^{\infty}(T)$ is the essential supremum of $|f|$ on $T$. The collection of all bounded holomorphic functions in the open unit disc $U$ forms a Banach algebra which can be identified (via radial limits) with the norm-closed subalgebra $H^{\infty}$ of $L^{\infty}(T)$.

A function $f$ in $L^{\infty}(T)$ is unimodular if $|f|=1$ a.e., on $T$. The inner functions are the unimodular members of $H^{\infty}$. It is well known that they play an important role in the study of $H^{\infty}$.

The main result (Theorem 1 ) is that the set of quotients of inner functions is norm-dense in the set of unimodular functions in $L^{\infty}(T)$. One consequence of this (Theorem 7 ) is that the set of radial limits of holomorphic functions of bounded characteristic in $U$ is norm-dense in $L^{\infty}(T)$. It is also shown (Theorem 3,4) that the Gelfand transforms of the inner functions separate points on the Silov boundary of $H^{\infty}$, and this is used to obtain a new proof (and generalization) of a theorem of D. J. Newman (Theorem 4).

Our proof of the main result uses only one nontrivial property of $H^{\infty}$, beyond the fact that $H^{\infty}$ is a norm-closed subalgebra of $L^{\infty}$. It therefore applies, without any extra effort, to a much more general situation which we now describe.

Let now $L^{\infty}$ denote the Banach algebra of all bounded measurable functions on some measure space $X$, normed by the essential supremum, and let $B$ be a norm-closed subalgebra of $L^{\infty}$. We say that $B$ has the annulus property if the following is true:

If $X$ is the union of disjoint measurable sets $E_{1}$ and $E_{2}$ and if $0<r_{1}<r_{2}<\infty$, then there exists $h$ in $B$ such that

(1) $1 / h$ is in $B$, and

(2) $|h|=r_{i}$ a.e., on $E_{i}$, for $i=1,2$.

That $H^{\infty}$ (in the classical setting described above) has the annulus property is well known: to see it, put $u=r_{i}$ on $E_{i}$ (now $T=E_{1} \cup E_{2}$ ), and define

$$
h(z)=\exp \left\{\frac{1}{2 \pi} \int_{-\pi}^{\pi} \frac{e^{i \theta}+z}{e^{i \theta}-z} \log u\left(e^{i \theta}\right) d \theta\right\} \quad(z \in U)
$$

Then $h$ maps $U$ into the annulus $\left\{w: r_{1}<|w|<r_{2}\right\}$, and the radial limits of $h$ have modulus $r_{i}$ a.e., on $E_{i}$. 
Furthermore, the $H^{\infty}$-algebras associated with weak*-Dirichlet algebras also have the annulus property. This is a special case of Lemma 2.4 .3 of [11]. We shall have no opportunity to use any other property of these algebras, and will therefore not even define them here. An excellent account of them is given in [11].

In order to avoid repetition we now state what our standing assumptions will be. Theorems 1 to 5 will deal with the general situation just described. $H^{\infty}$ will simply denote some subalgebra of some $L^{\infty}$, the only other hypothesis being that $H^{\infty}$ has the annulus property. The "inner functions" will again be the unimodular members of $H^{\infty}$. Theorems $6,7,8$ are more special and deal with the classical situation of the unit circle.

THEOREM 1. The set of all quotients of inner functions is normdense in the set of all unimodular functions in $L^{\infty}$.

Proof. Since the measurable unimodular functions taking finitely many values are norm-dense in the set of all unimodular functions in $L^{\infty}$, and since each function of the latter type is a product of finitely many unimodular functions each taking at most two values, it is sufficient to prove the following.

Proposition. If $E_{1}$ and $E_{2}$ are disjoint measurable subsets of $X$ whose union is $X$, if $\lambda_{1}$ and $\lambda_{2}$ are complex numbers of modulus 1 , and if $\varepsilon>0$, then there exist inner functions $\phi_{1}$ and $\phi_{2}$ such that

$$
\left|\lambda_{i}-\phi_{1}(x) / \dot{\phi}_{2}(x)\right|<\varepsilon \quad \text { a.e., on } E_{i}(i=1,2) \text {. }
$$

It involves no loss of generality to assume that $\lambda_{1} \neq \lambda_{2}$. Let $\alpha_{1}$ and $\alpha_{2}$ be closed disjoint subares of $T$, of length less than $\varepsilon$, containing $\lambda_{1}$ and $\lambda_{2}$, respectively. Let $\Omega$ be the complement of $\alpha_{1} \cup \alpha_{2}$ in the Riemann sphere. Then there is an annulus

$$
D=\left\{z: r_{1}<|z|<r_{2}\right\}
$$

and a continuous function $\Phi$ on its closure $\bar{D}$ whose restriction to $D$ is a one-to-one conformal map of $D$ onto $\Omega$ ([2], p. 247). If $|z|=r_{i}$ then $\Phi(z)$ is in $\alpha_{i}(i=1,2)$. The reflection principle shows that $\Phi$ is holomorphic on $\bar{D}$, except for a simple pole at some point $z_{0}$ in $D$. By a theorem of Ahlfors [1] there exists a function $\Phi_{2}$, holomorphic on $\bar{D}$, such that $\Phi_{2}$ has a zero at $z_{0}$ and $\left|\Phi_{2}(z)\right|=1$ on $\partial D$. Define $\Phi_{1}=$ $\Phi \cdot \Phi_{2}$. Then $\Phi_{1}$ is holomorphic on $\bar{D},\left|\Phi_{1}(z)\right|=1$ on $\partial D$, and $\Phi=\Phi_{1} / \Phi_{2}$.

By the annulus property which $H^{\infty}$ satisfies, there exists $h$ in $H^{\infty}$ such that $|h|=r_{i}$ a.e., on $E_{i}$, and $1 / h$ is in $H^{\infty}$. Thus $h$ maps $X$ into $\partial D,\|h\|_{\infty}=r_{2}$ and $\|1 / h\|_{\infty}=1 / r_{1}$. Since $\Phi_{1}$ and $\Phi_{2}$ are holomor- 
phic on $\bar{D}$, their Laurent expansions converge uniformly on $\bar{D}$. Since $H^{\infty}$ is norm-closed, this implies that the compositions $\phi_{1}=\Phi_{1} \circ h$ and $\phi_{2}=\Phi_{2} \circ h$ are in $H^{\infty}$. Clearly, they are also inner. Finally, $\phi_{1} / \phi_{2}=$ $\Phi \circ h$, and $(\Phi \circ h)(x)$ is in $\alpha_{i}$ for almost every $x$ in $E_{i}(i=1,2)$.

This proves the proposition, and hence Theorem 1 .

THEOREM 2. Let $Q$ be the set of all functions of the form $\psi \bar{\phi}$, where $\psi$ is a finite linear combination of inner functions and $\phi$ is inner. Then $Q$ is norm-dense in $L^{\infty}$.

Proof. By Theorem 1, the norm-closure $\bar{Q}$ of $Q$ contains all unimodular functions in $L^{\infty}$. Let $\chi_{E}$ be the characteristic function of a measurable set $E \subset X$. Note that $2 \chi_{E}-1$ is unimodular, and hence is in $\bar{Q}$. Since $\bar{Q}$ is a linear space, it follows that $\chi_{E}$ is in $\bar{Q}$ for every measurable $E \subset X$, and hence $\bar{Q}=L^{\infty}$.

Since $Q$ is the algebra generated by the inner functions and their complex conjugates, Theorem 2 may be restated as follows:

COROLlaRY. The self-adjoint algebra generated by the inner functions is norm-dense in $L^{\infty}$.

REMARK. The subgroup $G$ consisting of those unimodular functions which are quotients of inner functions has already occurred in certain studies ([5], [7, p. 12]). Theorem 1 shows how delicate the question of membership in $G$ is. Note that $G \subset Q$ (see Theorem 2) and that $Q \subset \widetilde{Q}$, where $\widetilde{Q}$ denotes the set of those functions in $L^{\infty}$ which are of the form $\phi \bar{\psi}$, where $\phi$ and $\psi$ are in $H^{\infty}$. In the classical situation, every nonconstant $f$ in $\widetilde{Q}$ satisfies

$$
\int_{T} \log |f| d m>-\infty \text {. }
$$

We doubt that this necessary condition is also sufficient (even for unimodular $f$ ) but we have no counterexample.

In connection with Theorem 2 , we recall that it is still an open question whether the closure $J$ of the set of finite linear combinations of inner functions is $H^{\infty}$ (cf. [3], p. 348). Actually, $J$ is a subalgebra of $H^{\infty}$ which in the classical case of the circle has the same maximal ideal space and Šilov boundary as $H^{\infty}$ (see the footnote to Theorem 3 and the proof of Theorem 4).

We now consider the maximal ideal space $M$ of $H^{\infty}$. The annulus property implies that 1 is in $H^{\infty}$, so $M$ is compact. The Gelfand transform $\hat{f}$ of an $f$ in $H^{\infty}$ is a continuous function of $M$, such that $\|\hat{f}\|=\|f\|_{\infty}$, where $\|\hat{f}\|$ denotes the maximum of $|\hat{f}|$ on $M$, and $\|f\|_{\infty}$ is the essential supremum of $|f|$ on $X$. We shall use the following 
notations:

If $\phi$ is inner, then

$$
K_{\phi}=\{\gamma \in M:|\hat{\phi}(\gamma)|=1\} .
$$

If $\Sigma$ is a set of inner functions, then

$$
K_{\Sigma}=\bigcap_{\phi \in \Sigma} K_{\phi} .
$$

If $\Sigma$ is the set of all inner functions in $H^{\infty}$, we write $K$ in place of $K_{\Sigma}$.

The Šilov boundary of $H^{\infty}$ will be denoted by $\partial$.

THEOREM 3. The Gelfand transforms of the inner functions separate points on $K .^{(1)}$

Proof. Let $\gamma_{0}$ and $\gamma_{1}$ be distinct points of $K$. There exists $f$ in $H^{\infty}$ with $\hat{f}\left(\gamma_{0}\right)=0$ and $\hat{f}\left(\gamma_{1}\right)=1$. By Theorem 2 , one can find $\dot{\phi}$ and $\psi$ such that $\phi$ is inner, $\psi$ is a finite linear combination of inner functions, and $\|\phi f-\psi\|_{\infty}<1 / 3$. Hence

$$
|\hat{\phi}(\gamma) \hat{f}(\gamma)-\hat{\psi}(\gamma)|<\frac{1}{3}
$$

for every $\gamma \in M$, in particular for $\gamma_{0}$ and $\gamma_{1}$. So $\left|\hat{\psi}\left(\gamma_{0}\right)\right|<1 / 3$, and $\left|\hat{\psi}\left(\gamma_{1}\right)\right|>2 / 3$ since $\left|\hat{\phi}\left(\gamma_{1}\right)\right|=1$. This shows that $\hat{\psi}$ separates $\gamma_{0}$ and $\gamma_{1}$.

Theorem 3 leads directly to a generalization of a theorem which D. J. Newmann proved in the classical case [9] and which characterizes the Silov boundary $\partial$ of $H^{\infty}$ in terms of inner functions:

\section{THEOREM 4. $\partial=K$.}

Proof. Let $\phi$ be inner. Choose $f$ in $H^{\infty}$, not identically 0 . Since $|\phi|=1$ on $X,\|f \phi\|_{\infty}=\|f\|_{\infty}=\|\hat{f}\|$. There exists $\gamma_{0}$ in $M$ at which $|\hat{f} \hat{\phi}|$ attains its maximum, $\|f \phi\|_{\infty}$, so that

$$
\|\hat{f}\|=\left|\hat{f}\left(\gamma_{0}\right) \hat{\phi}\left(\gamma_{0}\right)\right| \leqq\|\hat{f}\| \circ\left|\hat{\phi}\left(\gamma_{0}\right)\right| \leqq\|\hat{f}\| \text {. }
$$

This implies that $\left|\hat{\phi}\left(\gamma_{0}\right)\right|=1$ (i.e., $\gamma_{0}$ is in $K_{\phi}$ ) and that $\left|\hat{f}\left(\gamma_{0}\right)\right|=\|\hat{f}\|$. Thus every $|\hat{f}|$ attains its maximum (relative to $M$ ) at some point of $K_{\phi}$. This says: $\partial \subset K_{\phi}$. Since $K$ is the intersection of all $K_{\phi}$, we have $\partial \subset K$.

To prove that $\partial$ fills all $K$, let $E$ be a proper compact subset of $K$, choose $\gamma_{1}$ in $K$ but not in $E$. It then follows from Theorem 3 that

1 Kenneth Hoffman has communicated to us a proof which together with Theorem 3 shows that in the classical case of the circle the inner functions separate points on all of $M$. 
there exist finitely many inner functions, say $\phi_{1}, \cdots, \phi_{n}$, such that $\phi_{i}\left(\gamma_{1}\right)=1$ for $1 \leqq i \leqq n$, but

$$
\inf _{i} \operatorname{Re} \hat{\phi}_{i}(\gamma)<1 \text { for every } \gamma \text { in } E \text {. }
$$

Then $f=1+\phi_{1}+\cdots+\phi_{n}$ is in $H^{\infty}, \hat{f}\left(\gamma_{1}\right)=n+1=\|\hat{f}\|$, but $|\hat{f}(\gamma)|<$ $n+1$ for every $\gamma$ in $E$. Hence $E$ does not contain $\partial$. This completes the proof.

The following result about function algebras was stated without proof in [4] by the first author. We point out that it does not depend on the annulus property.

LEMMA. Let $\Sigma$ be a multiplicative semigroup of inner functions. Let $\mathfrak{A}_{\Sigma}$ be the norm-closed subalgebra of $L^{\infty}$ which is generated by $H^{\infty}$ and the complex conjugates of the members of $\Sigma$. Then the maximal ideal space $M_{\Sigma}$ of $\mathfrak{A}_{\Sigma}$ can be identified with the set $K_{\Sigma} \subset M$.

Proof. Let $\Gamma$ be a multiplicative linear functional on $\mathfrak{A}_{\Sigma}$. Restricting $\Gamma$ to $H^{\infty}$, we see that to each such $\Gamma$ corresponds a unique $\gamma$ in $M$, denoted by $\tau(\Gamma)$, such that $\Gamma(f)=\hat{f}(\gamma)$ for all $f$ in $H^{\infty}$.

Suppose $\gamma=\tau(\Gamma)$ and $\phi$ is in $\Sigma$. Since $\phi \bar{\phi}=1$, we have

$$
\Gamma(\bar{\phi})=\Gamma\left(\phi^{-1}\right)=1 / \Gamma(\phi)=1 / \hat{\phi}(\gamma) .
$$

This shows that $\Gamma$ is determined by $\gamma$, so $\tau: M_{\Sigma} \rightarrow M$ is one-to-one. It is easy to see that $\tau$ is continuous. Since both spaces are compact and Hausdorff, $\tau$ is a homeomorphism. Furthermore, $\tau\left(M_{\Sigma}\right) \subset K_{\Sigma}$, for if $\gamma=\tau(\Gamma)$, then $|\hat{\phi}(\gamma)| \leqq\|\phi\|_{\infty}=1$, and also

$$
|1 / \hat{\phi}(\gamma)|=|\Gamma(\bar{\phi})| \leqq\|\bar{\phi}\|_{\infty}=1,
$$

so that $|\hat{\phi}(\gamma)|=1$ for every $\phi$ in $\Sigma$ and every $\gamma$ in $\tau\left(M_{\Sigma}\right)$.

We want to prove that $\tau\left(M_{\Sigma}\right)=K_{\Sigma}$. To do this, we fix $\gamma$ in $K_{\Sigma}$, and show that $\gamma$ is in $\tau\left(M_{\Sigma}\right)$.

For $\psi$ in $H^{\infty}$ and $\phi$ in $\Sigma$, define

$$
\Gamma_{0}(\psi \bar{\psi} \bar{\phi})=\hat{\psi}(\gamma) / \hat{\phi}(\gamma)
$$

If $\psi_{1} \bar{\phi}_{1}=\psi_{2} \bar{\phi}_{2}$, then $\psi_{1} \phi_{2}=\psi_{2} \phi_{1}$, which implies $\hat{\psi}_{1}(\gamma) \hat{\phi}_{2}(\gamma)=\hat{\psi}_{2}(\gamma) \hat{\phi}_{1}(\gamma)$, and since $\gamma$ is in $K_{\Sigma}$, it follows that $\Gamma_{0}\left(\psi_{1} \bar{\phi}_{1}\right)=\Gamma_{0}\left(\psi_{2} \bar{\phi}_{2}\right)$. In otherwords, $\Gamma_{0}$ is well defined on a dense subalgebra of $\mathfrak{A}_{\Sigma}$. It is easy to check that $\Gamma_{0}$ is linear and multiplicative on this subalgebra. Finally (using the fact that $\gamma$ is in $K_{\Sigma}$ once more),

$$
\left|\Gamma_{0}(\psi \bar{\psi})\right|=|\hat{\psi}(\gamma) / \hat{\phi}(\gamma)|=|\hat{\psi}(\gamma)| \leqq\|\psi\|_{\infty}=\|\psi \bar{\phi}\|_{\infty},
$$


so that $\Gamma_{0}$ is bounded and can therefore be extended to a multiplicative linear functional $\Gamma$ on $\mathfrak{A}_{\Sigma}$. It is clear that $\tau(\Gamma)=\gamma$, and the proof is complete.

As a consequence, we obtain a theorem of I. J. Schark ([10], [8, p. 174]) which Srinivasan and Wang [11, p. 232] have extended to the context of Weak*-Dirichlet algebras:

THEOREM 5. The Silov boundary $\partial$ of $H^{\infty}$ can be identified with the maximal ideal space $M_{\infty}$ of $L^{\infty}$.

Proof. Let $\Sigma$ be the set of all inner functions. Then

$$
\hat{\partial}=K=K_{\Sigma}=M_{\Sigma}=M_{\infty} \text {. }
$$

The first of these equalities is Theorem 4, the second is the definition of $K$, the third is the preceding lemma, and the fourth follows from Theorem 2, since the latter asserts that $\mathfrak{A}_{\Sigma}=L^{\infty}$.

We now return to the classical situation, i.e., to the unit circle. Recall that an inner function in the open unit disc $U$ is said to be singular if it has no zero in $U$.

Theorem 6. Suppose $f$ is in $L^{\infty}(T),|f|=1,0<\varepsilon<1$.

(a) There exist Blaschke products $B_{1}$ and $B_{2}$ such that

$$
\left\|f-B_{1} / B_{2}\right\|_{\infty}<\varepsilon \text {. }
$$

(b) There exist inner functions $\dot{\phi}_{1}$ and $\dot{\phi}_{2}$, with $\dot{\phi}_{2}$ singular such that

$$
\left\|f-\dot{\phi}_{1} / \dot{\phi}_{2}\right\|_{\infty}<\varepsilon
$$

Of course, the expression $B_{1} / B_{2}$ in (a) refers to the radial limit function of the quotient of the two Blaschke products, and the norm is the essential supremum over $T$.

Proof. (a) is an immediate consequence of Theorem 1, because of Frostman's Theorem ([6, pp. 112-113], [8, p. 175]) which asserts that the Blaschke products are norm-dense in the set of all inner functions.

By Theorem 1, it suffices to prove (b) for the case $f=1 / \psi$, where $\psi$ is inner. Define

$$
u(w)=\exp \left\{c \frac{w+1}{w-1}\right\}
$$

where $c>0$ is so chosen that $3 u(0)<\varepsilon$, and put 


$$
u_{1}(w)=\frac{u(w)-u(0)}{w[1-u(0) u(w)]} .
$$

Then $u_{1}$ is inner, and one checks easily that

$$
\left|u(w)-w u_{1}(w)\right|<\varepsilon \quad(w \in U) .
$$

Put $w=\psi(z)$ in this inequality, define $\phi_{1}=u_{1} \circ \psi$ and $\phi_{2}=u \circ \psi$. Then $\phi_{1}$ and $\phi_{2}$ are inner, $\phi_{2}$ has no zero in $U$, and

$$
\left|\dot{\phi}_{2}(z)-\psi(z) \dot{\phi}_{1}(z)\right|<\varepsilon \quad(z \in U) .
$$

To complete the proof, take radial limits in the last inequality and divide by $\psi \phi_{2}$.

Because of Theorem $6(\mathrm{~b})$, Theorem 2 now takes the following form:

THEOREM 7. If $f$ is in $L^{\infty}(T)$ and $\varepsilon>0$, then there is a singular inner function $\phi$ and a finite linear combination $\psi$ of inner functions, such that

$$
\|f-\psi / \phi\|_{\infty}<\varepsilon
$$

Note that $\psi / \phi$ is a holomorphic function in $U$, of bounded characteristic (being a quotient of two $H^{\circ}$-functions). Thus the radial limits of holomorphic functions of bounded characteristic are norm-dense in $L^{\infty}(T)$.

We conclude with the observation that the set $K$ which was described prior to Theorem 3 can be defined (in the classical case) by means of the singular inner functions alone:

THEOREM 8. If $\gamma$ in $M$ is such that $|\hat{\psi}(\gamma)|<1$ for some inner function $\psi$, then there is a singular inner function $\phi$ with $|\hat{\phi}(\gamma)|<1$.

Proof. By Theorem $6(\mathrm{~b})$, with $\varepsilon=1-|\hat{\psi}(\gamma)|$, there are inner functions $\phi_{1}$ and $\phi_{2}$, with $\phi_{2}$ singular, such that

$$
\left|\hat{\phi}_{2}(\gamma)-\hat{\psi}(\gamma) \hat{\phi}_{1}(\gamma)\right| \leqq\left\|\phi_{2}-\psi \phi_{1}\right\|_{\infty}<1-|\hat{\psi}(\gamma)|,
$$

which implies that

$$
\left|\hat{\phi}_{2}(\gamma)\right|<\left|\hat{\psi}(\gamma) \hat{\phi}_{1}(\gamma)\right|+1-|\hat{\psi}(\gamma)| \leqq 1 .
$$

Theorem 8 adds an eighth equivalent condition to the seven that are listed on p. 179 of [8]. 


\section{BIBLIOGRAPHY}

1. L. V. Ahlfors, Bounded analytic functions, Duke Math. J. 14 (1947), 1-11.

2. - Complex analysis, 2nd Ed., McGraw-Hill, New York, 1966.

3. F. T. Birtel, ed., Function algebras, Scott, Foresman, Glenview, Ill., 1966.

4. R. G. Douglas, Toeplitz and Wiener-Hopf operators in $H^{\infty}+C$, Bull. Amer. Math. Soc. 74 (1968), 895-899.

5. R. G. Douglas, H. S. Shapiro, and A. L. Shields, On cyclic vectors of the backward shift, Bull. Amer. Math. Soc. 73 (1967), 156-159.

6. O. Frostman, Potentiel d'équilibre et capacité des ensembles, Lunds Univ. Mat. Sem. 3 (1935).

7. H. Helson, Invariant subspaces, Academic Press, New York, 1964.

8. K. Hoffman, Banach spaces of analytic functions, Prentice Hall, Englewood Cliffs, N. J., 1962.

9. D. J. Newman, Interpolation in $H^{\infty}$, Trans. Amer. Math. Soc. 92 (1959), 438-445. 10. I. J. Schark, The maximal ideals in an algebra bounded analytic functions, J. Math. Mech. 10 (1961), 735-746.

11. T. P. Srinivasan and J.-K. Wang, Weak*-Dirichlet algebras, pp. 216-249 in [3].

Received March 10, 1969. The First author is a Fellow of the Alfred P. Sloan Foundation. Research supported in part by an NSF Grant GP-8310. The second author was partially supported by NSF Grant GP-6764.

UNIVERSITY OF MICHIGAN AND

UNIVERSITY OF WISCONSIN 


\section{PACIFIC JOURNAL OF MATHEMATICS}

\section{EDITORS}

\author{
H. ROYDEN \\ Stanford University \\ Stanford, California \\ Richard Pierce \\ University of Washington \\ Seattle, Washington 98105
}

\author{
J. DugundJI \\ Department of Mathematics \\ University of Southern California \\ Los Angeles, California 90007 \\ BASIL GoRDON \\ University of California \\ Los Angeles, California 90024
}

\section{ASSOCIATE EDITORS}
E. F. BECKENBACH
B. H. Neumann
F. WOLF
K. YoSHIDA

\section{SUPPORTING INSTITUTIONS}

\author{
UNIVERSITY OF BRITISH COLUMBIA \\ CALIFORNIA INSTITUTE OF TECHNOLOGY \\ UNIVERSITY OF CALIFORNIA \\ MONTANA STATE UNIVERSITY \\ UNIVERSITY OF NEVADA \\ NEW MEXICO STATE UNIVERSITY \\ OREGON STATE UNIVERSITY \\ UNIVERSITY OF OREGON \\ OSAKA UNIVERSITY \\ UNIVERSITY OF SOUTHERN CALIFORNIA
}

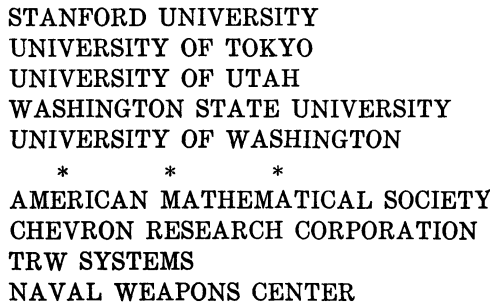

The Supporting Institutions listed above contribute to the cost of publication of this Journal, but they are not owners or publishers and have no responsibility for its content or policies.

Mathematical papers intended for publication in the Pacific Journal of Mathematics should be in typed form or offset-reproduced, double spaced with large margins. Underline Greek letters in red, German in green, and script in blue. The first paragraph or two must be capable of being used separately as a synopsis of the entire paper. It should not contain references to the bibliography. Manuscripts, in duplicate if possible, may be sent to any one of the four editors. Please classify according to the scheme of Math. Rev. 36, 1539-1546. All other communications to the editors should be addressed to the managing editor, Richard Arens, University of California, Los Angeles, California, 90024.

50 reprints are provided free for each article; additional copies may be obtained at cost in multiples of 50 .

The Pacific Journal of Mathematics is published monthly. Effective with Volume 16 the price per volume (3 numbers) is $\$ 8.00$; single issues, $\$ 3.00$. Special price for current issues to individual faculty members of supporting institutions and to individual members of the American Mathematical Society: $\$ 4.00$ per volume; single issues $\$ 1.50$. Back numbers are available.

Subscriptions, orders for back numbers, and changes of address should be sent to Pacific Journal of Mathematics, 103 Highland Boulevard, Berkeley, California, 94708.

PUBLISHED BY PACIFIC JOURNAL OF MATHEMATICS, A NON-PROFIT CORPORATION

Printed at Kokusai Bunken Insatsusha (International Academic Printing Co., Ltd.), 7-17, Fujimi 2-chome, Chiyoda-ku, Tokyo, Japan. 


\section{Pacific Journal of Mathematics}

\section{Vol. 31, No. $2 \quad$ December, 1969}

Efraim Pacillas Armendariz, Quasi-injective modules and stable torsion

classes..........................................

J. Adrian (John) Bondy, On Ulam's conjecture for separable graphs...

Vasily Cateforis and Francis Louis Sandomierski, On commutative rings over which the singular submodule is a direct summand for every module .....

Rafael Van Severen Chacon, Approximation of transformations with continuous

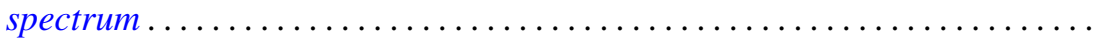

Raymond Frank Dickman and Alan Zame, Functionally compact spaces ...... 303

Ronald George Douglas and Walter Rudin, Approximation by inner

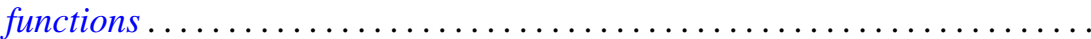

John Walter Duke, A note on the similarity of matrix and its conjugate

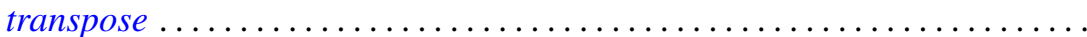

Micheal Neal Dyer and Allan John Sieradski, Coverings of mapping

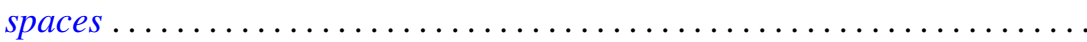

Donald Campbell Dykes, Weakly hypercentral subgroups of finite groups .....

Nancy Dykes, Mappings and realcompact spaces.....................

Edmund H. Feller and Richard Laham Gantos, Completely injective

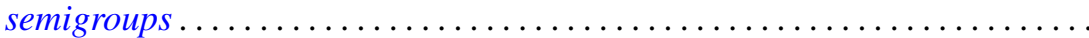

Irving Leonard Glicksberg, Semi-square-summable Fourier-Stieltjes

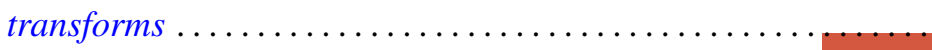

Samuel Irving Goldberg and Kentaro Yano, Integrability of almost cosymplectic structures...

Seymour Haber and Charles Freeman Osgood, On the sum $\sum\langle n \alpha\rangle^{-t}$ and numerical integration ..........................

Sav Roman Harasymiv, Dilations of rapidly decreasing functions ....

William Leonard Harkness and R. Shantaram, Convergence of a sequence of

transformations of distribution functions

Herbert Frederick Kreimer, Jr., A note on the outer Galois theory of rings ...

James Donald Kuelbs, Abstract Wiener spaces and applications to analysis. .

Roland Edwin Larson, Minimal $T_{0}$-spaces and minimal $T_{D}$-spaces...

A. Meir and Ambikeshwar Sharma, On Ilyeff's conjecture .

Isaac Namioka and Robert Ralph Phelps, Tensor products of compact convex sets....

James L. Rovnyak, On the theory of unbounded Toeplitz operators ....

Benjamin L. Schwartz, Infinite self-interchange graphs.......

George Szeto, On the Brauer splitting theorem...

Takayuki Tamura, Semigroups satisfying identity $x y=f(x$,

Kenneth Tolo, Factorizable semigroups .................. 